\title{
Scaling tests for overlap fermions
}

Jan Wennekers* and Hartmut Wittig

DESY, Theory Group, Notkestraße 85, D-22603 Hamburg, Germany

E-mail: jan.wennekers@desy.de, hartmut.wittig@desy.de

We present results on the scaling behaviour of mesonic observables with Ginsparg-Wilson fermions in quenched QCD. Using the overlap operator we compute two-point correlation functions on four lattices with $5.8458 \leq \beta \leq 6.1366$. We perform continuum extrapolations for the pseudoscalar decay constant and the vector mass.

Furthermore we compute the non-perturbative renormalization of the scalar density. We interpolate our data to the physical kaon mass and and match it with continuum results for $\mathrm{O}(a)$-improved Wilson fermions. With this method we are able to obtain $Z_{\mathrm{S}}$ over a range of lattice spacings and extrapolate the renormalized quark condensate to the continuum limit.

A full account of our results can be found in [1].

[1] J. Wennekers and H. Wittig, On the renormalized scalar density in quenched QCD, JHEP 09 (2005) 059, [hep-lat/0507026].

XXIIIrd International Symposium on Lattice Field Theory

25-30 July 2005

Trinity College, Dublin, Ireland

*Speaker. 